\title{
MODELAGEM E SIMULAÇÃO DO PROCESSO DE DESIDRATAÇÃO DE BIOETANOL A ETENO
}

\author{
J. G. S. S. MAIA ${ }^{1}$, R.S. OLIVEIRA ${ }^{2}$, A. R. SECCHI ${ }^{3}$, E. C. BISCAIA Jr ${ }^{4}$ \\ 1,2,3,4 Universidade Federal do Rio de Janeiro, COPPE, Programa de Engenharia Química \\ E-mail para contato: jeiveison@ peq.coppe.ufrj.br
}

\begin{abstract}
RESUMO - A produção de plásticos a partir de uma matéria-prima renovável é de grande interesse na atualidade. $\mathrm{O}$ uso de resíduos de biomassa à base de carbono na produção de plásticos pode atender parcialmente a crescente demanda por plásticos no futuro próximo. O etileno, monômero mais utilizado pela indústria de plástico, produzido pela desidratação catalítica do etanol, é um processo que estava estagnado nas últimas décadas devido à viabilidade e ao baixo custo do gás natural e da nafta, fazendo com que a produção de etileno ocorresse pelo craqueamento térmico destes hidrocarbonetos. $\mathrm{O}$ interesse pela produção de etileno a partir do bioetanol vem se renovando motivado principalmente pelo apelo ambiental e econômico. O principal objetivo deste trabalho é a construção de modelos matemáticos no simulador EMSO para a simulação e otimização da produção de eteno a partir da desidratação do etanol, com o intuito de melhorar o desempenho do processo. O modelo fenomenológico proposto é fundamentado nos balanços de massa, momentum e energia do processo. Os resultados alcançados são satisfatórios com a teoria presente na literatura.
\end{abstract}

\section{INTRODUÇÃO}

Existem várias matérias-primas renováveis para produção de plástico, mas apenas um número limitado de produtos petroquímicos poderia ser produzido a partir de biomassa por meio de tecnologias comerciais economicamente viáveis. O etileno é a matéria-prima mais utilizada pela indústria de plástico e pode ser produzido por tecnologias disponíveis, tais como a desidratação de bioetanol. A produção de eteno por desidratação catalítica de etanol é uma importante via alternativa, uma vez que o etanol pode ser obtido a partir de fontes renováveis. Um levantamento das possíveis reações na decomposição do etanol pode ser encontrado em Ávila Neto (2009).

No processo de desidratação do etanol, esse é vaporizado, aquecido em um forno e enviado a um reator de leito fixo. O processo pode operar em modo isotérmico (usando um fluido de aquecimento) ou em modo adiabático (usando vapor de diluição). O efluente do reator é resfriado para que a maior parte da água seja removida em uma torre de condensação. $\mathrm{O}$ eteno bruto sai no topo desta torre, sofre lavagens para remoção de ácidos e outros componentes solúveis em água e passa por um leito de secagem, gerando assim eteno de alta pureza. A remoção das impurezas remanescentes é feita por colunas de destilação das quais sai o eteno grau polímero que é enviado às plantas de polimerização (Morschbacker, 2009). 
Os modelos matemáticos apresentados neste trabalho são referentes ao reator de leito fixo presente no processo e são fundamentados nos balanços de massa, momentum e energia. Os modelos cinéticos são baseados em expressões de taxa provenientes da literatura referenciada, como, por exemplo, as presentes no trabalho de Kagyrmanova et al. (2011). A implementação dos modelos foi feita no software computacional Mathematica e no simulador de processos EMSO.

\section{MODELAGEM DO PROCESSO}

As principais hipóteses simplificadoras para a construção do modelo são: sistema pseudohomogêneo unidimensional com dispersão axial mássica e térmica; coeficiente efetivo de difusão mássica e de condutividade térmica constantes; operação adiabática; apenas a capacidade térmica da mistura reacional é considerada; atividade do catalisador constante; dissipação de calor pelas forças viscosas e pela difusão mássica desprezível; fluido newtoniano e viscosidade constante.

\subsection{Leis Fundamentais de Conservação}

Os princípios de conservação de massa global e por componente, energia e quantidade de movimento quando aplicados ao sistema dinâmico em estudo por meio do adimensionamento das variáveis independentes e dependentes por valores de referência, dados por:

$$
t=\frac{t^{\prime}}{L / v_{r e f}} ; \quad y=\frac{z}{L} ; \quad x_{i}=\frac{C_{i}}{C_{r e f}} ; \quad \theta=\frac{T}{T_{r e f}} ; \quad \Pi=\frac{P}{P_{r e f}} ; \quad \omega=\frac{v}{v_{r e f}} ; \quad \phi=\frac{\rho}{\rho_{\text {ref }}} .
$$

Resultam no sistema de equações algébrico-diferenciais implementadas:

$$
\begin{aligned}
& \frac{\partial \phi}{\partial t}+\frac{\partial(\omega \phi)}{\partial y}=0 \\
& \frac{\partial x_{i}}{\partial t}+\frac{\partial\left(\omega x_{i}\right)}{\partial y}=\frac{1}{P e_{M}} \frac{\partial^{2} x_{i}}{\partial y^{2}}+\sum_{j=1}^{N} v_{i, j} D a_{j} r_{j} \\
& \phi \frac{\partial \theta}{\partial t}+\omega \phi \frac{\partial \theta}{\partial y}=\frac{1}{P e_{H}} \frac{\partial^{2} \theta}{\partial y^{2}}+\sum_{j=1}^{N} B_{j} D a_{j} r_{j}+\mathbb{C}\left(\frac{\partial \Pi}{\partial t}+\omega \frac{\partial \Pi}{\partial y}\right) \\
& \phi \frac{\partial \omega}{\partial t}+\omega \phi \frac{\partial \omega}{\partial y}=-B K \omega-B P \phi \omega^{2}-E u \frac{\partial P}{\partial y}+\frac{1}{F r} \phi
\end{aligned}
$$

Essas equações têm como condições iniciais as seguintes relações:

$$
x_{i}(y, 0)=y_{i 0}(y) ; \quad \theta(y, 0)=\theta_{0}(z) ; \quad \Pi(y, 0)=\Pi_{0}(y) ; \quad \omega(y, 0)=\omega_{0}(y) .
$$

As condições de contorno para a concentração e para a temperatura, na entrada e na saída do reator são baseadas no trabalho de Langmuir (1908), deduzidas intuitivamente por Danckwerts (1953), cuja dedução rigorosa foi realizada por Bischoff (1961) e são dadas por: 
$x_{i f}=x_{i}(0, t)-\left.\frac{1}{P e_{M}} \frac{\partial x_{i}(y, t)}{\partial y}\right|_{y=0}$

$\theta_{f}=\theta(0, t)-\left.\frac{1}{P e_{H}} \frac{\partial \theta(y, t)}{\partial z}\right|_{y=0}$

$$
\left.\frac{\partial x_{i}(0, t)}{\partial y}\right|_{y=1}=0 ;\left.\frac{\partial \theta(y, t)}{\partial y}\right|_{y=1}=0
$$

As demais condições de contorno são: $\Pi(0, t)=\Pi_{f}(t) ; \quad \omega(0, t)=\omega_{f}(t)$.

Os parâmetros adimensionais obtidos estão apresentados na Tabela 1.

Tabela 1: Parâmetros adimensionais do modelo

\begin{tabular}{|c|c|c|c|}
\hline Nome & Símbolo & Definição & Descrição \\
\hline No de Péclet Mássico & $P e_{M}$ & $\frac{L v_{r e f}}{D_{M}}$ & Advecção vs, Difusão (mássica) \\
\hline No de Péclet Térmico & $P e_{H}$ & $\frac{L v_{r e f} \rho_{r e f} \hat{C}_{P, M}^{\circ}}{k_{H}}$ & Advecção vs, Difusão (térmica) \\
\hline $\mathrm{N}^{\mathrm{o}}$ de Damköhler* & $D a_{j}$ & $\frac{L}{v_{r e f}} A_{j} e^{-\frac{\gamma_{j}}{\theta}}\left(C_{r e f}\right)^{o d-1}$ & $\begin{array}{c}\text { Reacional vs. Transporte } \\
\text { (tempo) }\end{array}$ \\
\hline $\begin{array}{l}\text { Energia de ativação } \\
\text { adimensional }\end{array}$ & $\gamma_{j}$ & $\frac{E a_{j}}{R T_{r e f}}$ & - \\
\hline $\begin{array}{c}\text { Temperatura } \\
\text { adiabática adimens. }\end{array}$ & $B_{j}$ & $\frac{\left(-\Delta \bar{H}_{j}^{\circ}\right) C_{r e f}}{\rho_{r e f} \hat{C}_{P, M}^{\circ} T_{r e f}}$ & $\begin{array}{c}\text { Reacional vs. Capacidade } \\
\text { (térmica) }\end{array}$ \\
\hline $\mathrm{N}^{\mathrm{o}}$ de Froude & $\mathrm{Fr}$ & $\frac{v_{r e f}^{2}}{g_{z} L}$ & $\begin{array}{c}\text { Cinética vs. Gravitacional } \\
\text { (energia) }\end{array}$ \\
\hline $\mathrm{N}^{\mathrm{o}}$ de Euler & $E u$ & $\frac{P_{r e f} / \rho_{r e f}}{v_{r e f}^{2}}$ & Mecânica vs. Cinética (energia) \\
\hline Eq. de Blake-Kozeny & $B K$ & $150 \frac{(1-\varepsilon)^{2}}{\varepsilon^{3}} \frac{\mu}{\rho_{\text {ref }} v_{r e f} d_{p}} \frac{\mathrm{L}}{\mathrm{d}_{\mathrm{p}}}$ & Região Laminar \\
\hline Eq. de Burke-Plummer & $B P$ & $\frac{7}{4} \frac{(1-\varepsilon)}{\varepsilon^{3}} \frac{\mathrm{L}}{\mathrm{d}_{\mathrm{p}}}$ & Região Turbulenta \\
\hline- & $\mathbb{C}$ & $\frac{P_{r e f} / \rho_{\text {ref }}}{\hat{C}_{P, M}^{\circ} T_{\text {ref }}}$ & Mecânica vs. Térmica (energia) \\
\hline
\end{tabular}

\subsection{Modelagem da Cinética Química}

Os modelos cinéticos utilizados neste trabalho são baseados nos trabalhos de Kagyrmanova et al. (2011), cujos estudos experimentais revelaram que os principais produtos da desidratação de etanol sobre a superfície do catalisador à base de alumina, são eteno, éter dietílico, acetaldeído, hidrogênio e butenos de acordo com o seguinte esquema de reação: 


$$
\begin{aligned}
& \mathrm{C}_{2} \mathrm{H}_{5} \mathrm{OH} \rightleftharpoons \mathrm{C}_{2} \mathrm{H}_{4}+\mathrm{H}_{2} \mathrm{O} \\
& 2 \mathrm{C}_{2} \mathrm{H}_{5} \mathrm{OH} \rightleftharpoons\left(\mathrm{C}_{2} \mathrm{H}_{5}\right)_{2} \mathrm{O}+\mathrm{H}_{2} \mathrm{O} \\
& \mathrm{C}_{2} \mathrm{H}_{5} \mathrm{OH} \rightleftharpoons \mathrm{C}_{2} \mathrm{H}_{4} \mathrm{O}+\mathrm{H}_{2} \\
& \left(\mathrm{C}_{2} \mathrm{H}_{5}\right)_{2} \mathrm{O} \rightleftharpoons 2 \mathrm{C}_{2} \mathrm{H}_{4}+\mathrm{H}_{2} \mathrm{O} \\
& 2 \mathrm{C}_{2} \mathrm{H}_{4} \rightleftharpoons \mathrm{C}_{4} \mathrm{H}_{8}
\end{aligned}
$$

Em temperaturas acima de $400{ }^{\circ} \mathrm{C}$ alguns traços de metano, propeno, buteno, isobutanol, acetaldeído, óxido de carbono e dióxido de carbono foram observados. Com o objetivo de facilitar a notação a seguinte referência é utilizada:

$A=\mathrm{C}_{2} \mathrm{H}_{5} \mathrm{OH} ; \quad B=\mathrm{C}_{2} \mathrm{H}_{4} ; \quad C=\mathrm{H}_{2} \mathrm{O} ; \quad D=\left(C_{2} \mathrm{H}_{5}\right)_{2} \mathrm{O} ; \quad E=\mathrm{C}_{2} \mathrm{H}_{4} \mathrm{O} ; \quad F=\mathrm{H}_{2} ; \quad G=\mathrm{C}_{4} \mathrm{H}_{8}$.

A ordem global da reação direta $(o d)$ nas reações 1, 3 e 4 é igual a 1 e nas demais é igual a 2. As expressões cinéticas das reações mencionadas, adimensionais, e a dependência da velocidade específica de reação com a temperatura, usando a relação de Arrhenius, são:

$$
\begin{aligned}
& r_{1}=\left(x_{A}-\frac{1}{K_{1}}\left(\frac{C_{r e f}}{C^{\circ}}\right) x_{B} x_{C}\right) \\
& r_{2}=\left(x_{A}^{2}-\frac{1}{K_{2}} x_{C} x_{D}\right) \\
& r_{3}=\left(x_{A}-\frac{1}{K_{3}}\left(\frac{C_{r e f}}{C^{\circ}}\right) x_{E} x_{F}\right)
\end{aligned}
$$

$$
\begin{aligned}
& r_{4}=\left(x_{D}-\frac{1}{K_{4}}\left(\frac{C_{r e f}}{C^{\circ}}\right)^{2} x_{B}^{2} x_{C}\right) \\
& r_{5}=\left(x_{B}^{2}-\frac{1}{K_{5}}\left(\frac{C^{\circ}}{C_{r e f}}\right) x_{G}\right) \\
& k_{j, D}=A_{j} e^{-\frac{E a_{j}}{R T}}
\end{aligned}
$$

\subsection{Relações Termodinâmicas e Constitutivas}

A capacidade calorífica específica padrão à pressão constante da mistura está relacionada à temperatura e à composição da mistura reacional através da expressão:

$$
\hat{C}_{P, M}^{\circ}=\frac{\sum_{i=1}^{n} C_{i} \alpha_{i}+T \sum_{i=1}^{n} C_{i} \beta_{i}+T^{2} \sum_{i=1}^{n} C_{i} \gamma_{i}+T^{3} \sum_{i=1}^{n} C_{i} \delta_{i}}{\sum_{i=1}^{n} C_{i} M_{i}}
$$

A expressão para o cálculo da variação da entalpia padrão de reação molar é dada por:

$\Delta \bar{H}_{j}^{\circ}=\Delta \bar{H}_{j, T_{0}}^{\circ}+\int_{T_{0}}^{T} \Delta \bar{C}_{P, j}^{\circ} d T$

em que: $\Delta \bar{C}_{P, j}^{\circ}=\sum_{i=1}^{n} v_{i, j} \bar{C}_{P, i}^{\circ} \quad$ e $\quad \bar{C}_{P, i}^{\circ}=\alpha_{i}+\beta_{i} T+\gamma_{i} T^{2}+\delta_{i} T^{3}$.

Para o cálculo da constante de equilíbrio químico para a reação " $j$ ", tem-se:

$$
K_{j}=\exp \left(\frac{\Delta \bar{H}_{j, T_{0}}^{\circ}-\Delta \bar{G}_{j, T_{0}}^{\circ}}{R T_{0}}-\frac{\Delta \bar{H}_{j, T_{0}}^{\circ}}{R T}-\frac{1}{R T} \int_{T_{0}}^{T} \Delta \bar{C}_{P, j}^{\circ} d T+\frac{1}{R} \int_{T_{0}}^{T} \Delta \bar{C}_{P, j}^{\circ} \frac{d T}{T}\right)
$$


A equação de estado utilizada é a equação para o gás ideal, dada por:

$\rho=\frac{P M_{M}}{R T}$

$$
M_{M}=\frac{\sum_{i=1}^{n} C_{i} M_{i}}{\sum_{i=1}^{n} C_{i}}
$$

\section{SIMULAÇÃO DO PROCESSO}

Para a simulação foram usados os dados dimensionais e operacionais da planta piloto referente ao trabalho de Kagyrmanova et al. (2011). O sistema de equações obtidas foi resolvido por duas metodologias distintas, sendo uma para o modelo dinâmico e outra para o modelo estacionário. Para o modelo dinâmico foi utilizado o "métodos das linhas", em que a variável espacial foi discretizada por diferenças finitas centrais de quarta ordem em 40 pontos de malha e a etapa de integração foi feita a partir de um método tipo BDF utilizado o pacote SUNDIALS.

A derivada de primeira ordem foi então aproximada por:

$\frac{d \Psi\left(\mathrm{y}_{k}\right)}{d y} \cong \frac{\Psi\left(\mathrm{y}_{k-2}\right)-8 \Psi\left(\mathrm{y}_{k-1}\right)+8 \Psi\left(\mathrm{y}_{k+1}\right)-\Psi\left(\mathrm{y}_{k+2}\right)}{12 h}$

Para a derivada de segunda ordem foi usada a aproximação:

$\frac{d^{2} \Psi\left(\mathrm{y}_{k}\right)}{d y^{2}} \cong \frac{-\Psi\left(\mathrm{y}_{k-2}\right)+16 \Psi\left(\mathrm{y}_{k-1}\right)-30 \Psi\left(\mathrm{y}_{k}\right)+16 \Psi\left(\mathrm{y}_{k+1}\right)-\Psi\left(\mathrm{y}_{k+2}\right)}{12 h^{2}}$

O aumento do custo computacional na resolução do sistema pentadiagonal obtido, devido à discretização de quarta ordem, quando comparado ao sistema tridiagonal obtido via discretização de segunda ordem, mostrou-se ínfimo para uma mesma acurácia. Além disso, a magnitude do erro de quarta ordem é acentuadamente menor quando comparado à de segunda ordem. A aproximação da segunda derivada por quarta ordem apresenta um erro de $\frac{1}{90} h^{4} \frac{d^{6} \Psi\left(\mathrm{y}_{k}\right)}{d y^{6}}$ contra $\frac{1}{12} h^{2} \frac{d^{4} \Psi\left(\mathrm{y}_{k}\right)}{d y^{4}}$ para a segunda ordem, já a aproximação da derivada primeira por quarta ordem apresenta um erro de $\frac{1}{30} h^{4} \frac{d^{5} \Psi\left(\mathrm{y}_{k}\right)}{d y^{5}}$ contra $\frac{1}{6} h^{2} \frac{d^{3} \Psi\left(\mathrm{y}_{k}\right)}{d y^{3}}$ obtido na aproximação de segunda ordem (Fornberg, 1998).

Para o modelo estacionário, o problema de valor de contorno é adaptado para um problema de valor inicial através de uma mudança de variável e então o método de "Single Shooting" é usado, com a etapa de integração também feita a partir de um método tipo BDF utilizando o pacote SUNDIALS. Nos dois modelos obtidos o índice diferencial é igual a 1.

As figuras seguir apresentam os perfis espaciais em regime estacionário das variáveis dependentes e de alguns números adimensionais caracterizantes do sistema. Por meio da análise da Figura 1 é possível observar que as reações $R_{1}$ e $R_{2}$ apresentam magnitudes muito superiores às reações, $R_{3}, R_{4}$ e $R_{5}$, o que é confirmado ao se observar a Figura 3 e que está de acordo com a 
literatura referenciada. No início do leito a formação de éter etílico é maior que a de eteno, o que é explicado com base na comparação entre os valores dos números de Damköhler da primeira e da segunda reação, em que $D a_{2}$ é maior que $D a_{1}$ em quase todo o leito. Entretanto, a formação do produto principal torna-se maior que a do éter etílico logo após os primeiros $10 \%$ do comprimento do leito, observação justificada por diversos fatores, ente eles, pela decomposição desse subproduto em eteno pela reação $R_{4}$ que é praticamente irreversível. As condições operacionais da simulação também contribuem para este efeito, por meio de temperaturas elevadas, baixas pressões e concentrações de etanol, características que favorece a reação principal.

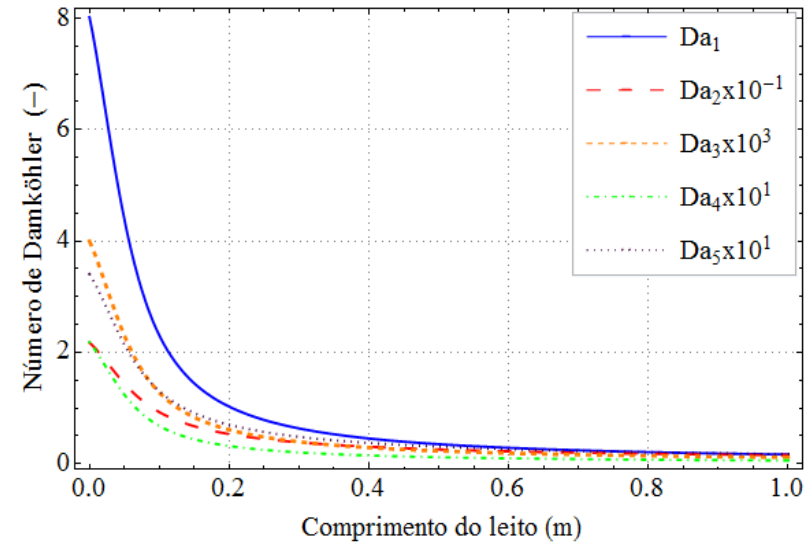

Figura 1 - Perfil espacial dos números de Damköhler.

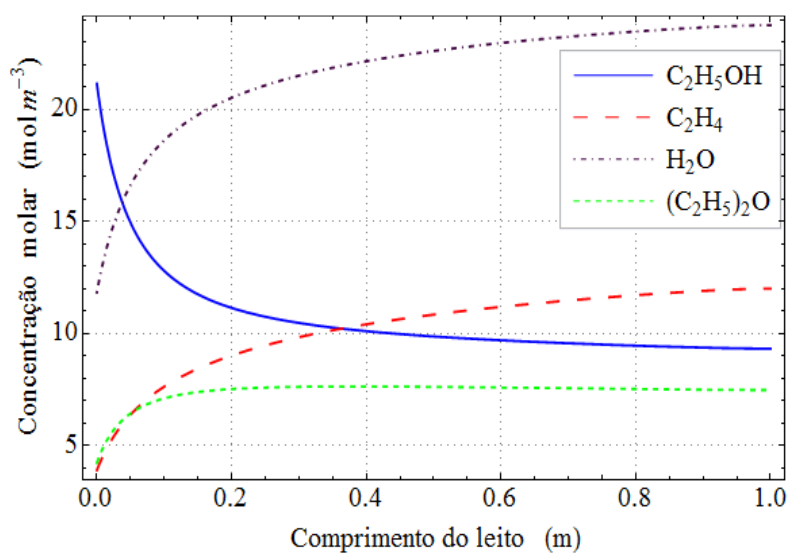

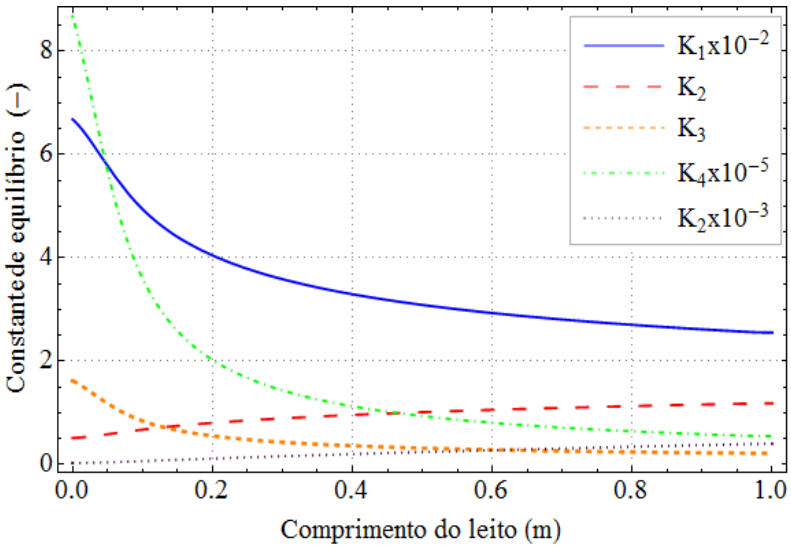

Figura 2 - Perfil espacial das constantes de equilíbrio químico.

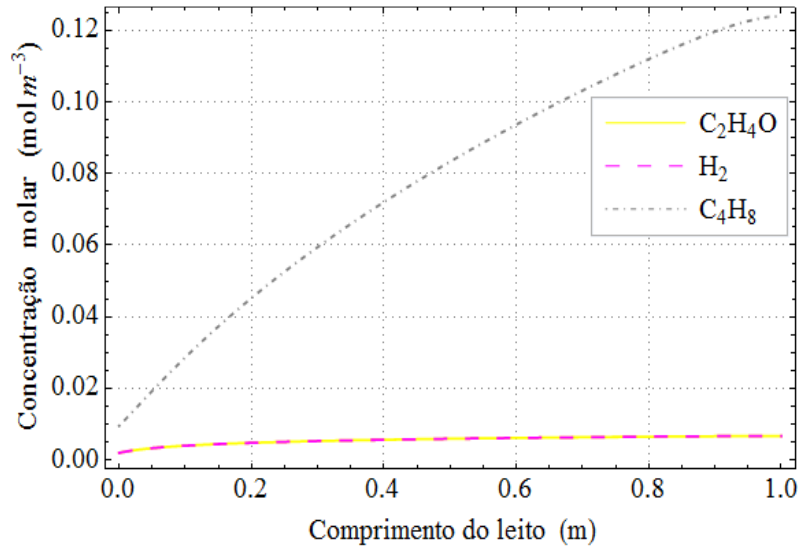

Figura 3 - Perfil espacial de concentração molar das espécies reacionais.

A partir da observação da Figura 2 verifica-se que apenas as reações $R_{2}$ e $R_{3}$ apresentam considerável reversibilidade. Essa é uma constatação importante, pois nessas reações o etanol é convertido em subprodutos de menor interesse. Além disso, nas reações $R_{1}$ e $R_{4}$, em que o eteno é formado, a reversibilidade dessas reações é praticamente nula. Analisando a natureza endotérmica das reações $R_{1}, R_{3}$ e $R_{4}$ e a natureza exotérmica das reações $R_{2}$ e $R_{5}$ e devido à diminuição da temperatura, o que pode ser observado na Figura 4, observa-se um caráter globalmente endotérmico do sistema. A energia de ativação da reação $R_{1}$ é maior do que a energia de ativação da reação $R_{2}$, logo uma maior temperatura favorecerá a diminuição dos efeitos desta diferença a favor da reação $R_{1}$. Também na Figura 4 é possível notar um perfil praticamente linear para a pressão, o que é comum em problemas com velocidades relativamente baixas. Examinando ainda 
a Figura 4 é constatada uma magnitude moderada, em torno de $2 \%$, para a queda de pressão. Essa constatação indica que o gradiente de pressão, presente no balanço de energia, pouco influência o perfil de temperatura neste sistema analisado. Na Figura 5 são apresentados os perfis de massa específica e de velocidade axial com variações inversas, o que é esperado, pois o produto entre essas duas variáveis deve ser constante ao longo de todo o leito no estado estacionário, caracterizando o não acúmulo de massa no leito. As variações presentes nessas duas variáveis foram da ordem de $5 \%$.

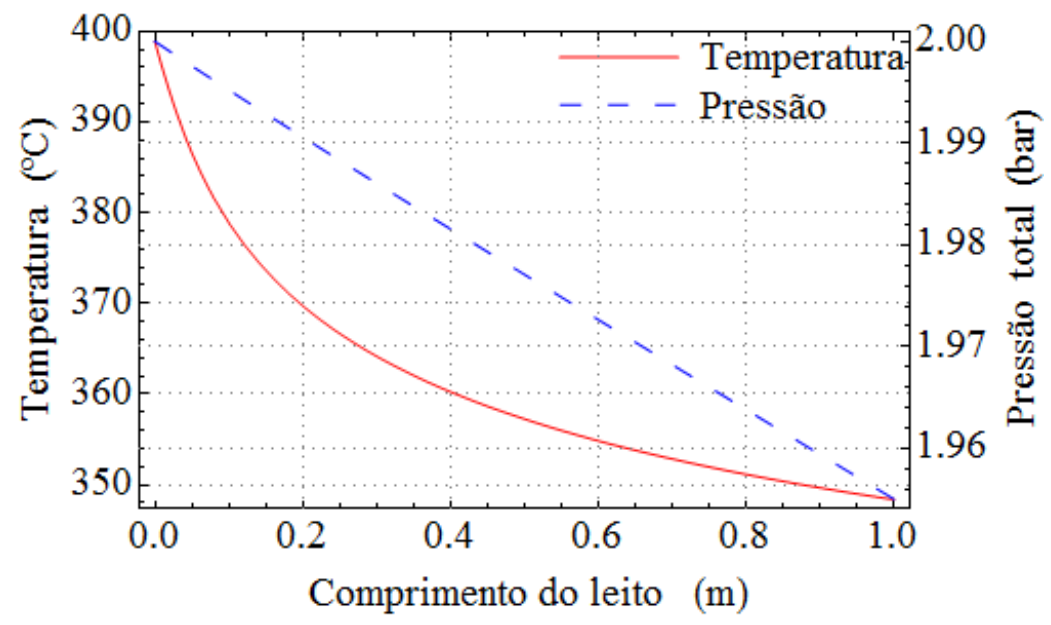

Figura 4 - Perfil espacial de temperatura e de pressão total.



Figura 5 - Perfil espacial da massa específica da mistura e da velocidade axial.

O número de Péclet mássico calculado com magnitude igual a 20 justifica a não simplificação da desconsideração do efeito da difusão mássica no sistema em análise. Porém, o valor encontrado para o Péclet térmico (em torno de 320) apresenta evidências que os efeitos térmicos de natureza difusiva, poderiam ser desconsiderados neste sistema em análise.

\section{CONCLUSÕES}

Neste trabalho, o processo catalítico da desidratação do bioetanol a etileno foi estudado por meio da modelagem hidrodinâmica e cinética e da simulação do processo, no qual a formação de etileno ocorre através de reações mistas. Os resultados encontrados estão de acordo com a literatura referenciada, demonstrando que o modelo proposto reproduz adequadamente o sistema 
estudado. A metodologia numérica utilizada se mostrou robusta e adequada. Decorrente dos bons resultados encontrados, o modelo pode então ser usado para a otimização das condições operacionais e as configurações do processo que maximizem a produção do produto principal, o eteno verde.

\section{NOMENCLATURA}

$A_{j}-$ fator pré-exponencial da reação $j \quad K_{j}$ - constante de equilíbrio químico da reação $j$

$\left(\left(\mathrm{mol} \mathrm{m} \mathrm{m}^{-3}\right)^{1-o d} \mathrm{~s}^{-1}\right)$;

$\bar{C}_{P, i}^{\circ}$ - capacidade calorífica padrão molar da espécie $i$ a pressão constante. $\left(\mathrm{mol}^{-1} \mathrm{~K}^{-1}\right)$;

$\hat{C}_{P, M}^{\circ}$ - capacidade calorífica padrão específica da mistura reacional a $\mathrm{P}$ const. $\left(\mathrm{Jg}^{-1} \mathrm{~K}^{-1}\right)$;

$C_{i}$ - concentração molar da espécie $i\left(\mathrm{~mol} \mathrm{~m}^{-3}\right)$;

$C^{\circ}$ - concentração molar padrão à $298 \mathrm{~K}$ e 1 bar $\left(\mathrm{mol} \mathrm{m}^{-3}\right)$;

$D_{M}-$ coeficiente efetivo de difusividade mássica $\left(m^{2} \cdot s^{-1}\right)$;

$d_{p}$ - diâmetro equivalente da partícula $(m)$;

$\Delta \bar{G}_{j, T_{0}}^{\circ}$ - variação da energia de Gibbs na reação $j$

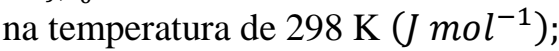

$\Delta \bar{H}_{j}^{\circ}$ - variação da entalpia padrão molar da reação $j\left(\mathrm{Jol}^{-1}\right)$;

$E a_{j}$ - Energia de ativação da reação $j\left(\mathrm{~J} \mathrm{~mol}^{-1}\right)$;

$\varepsilon$ - porosidade do leito;

$g_{z}$ - aceleração da gravidade na direção $\left(m^{-2}\right)$;

$L$ - comprimento total do leito $(m)$;

$k_{H}$ - coeficiente efetivo de condutividade térmica

$\left(W m^{-1} K^{-1}\right)$;

$k_{j, D}$ - velocidade específica da reação $j$ direta $\left(\left(\mathrm{mol} \mathrm{m} \mathrm{m}^{-3}\right)^{1-o d} \mathrm{~s}^{-1}\right)$;

$M_{i}$ - massa molar da espécie $i\left(\mathrm{~kg} \mathrm{~mol}^{-1}\right)$;

$M_{M}$ - massa molar da mistura reacional $\left(\mathrm{kg} \mathrm{mol}^{-1}\right)$;

$\mu$ - viscosidade dinâmica da mistura (Pa.s);

$v_{i, j}$ - coeficiente estequiométrico da espécie $i$ na reação $j$;

$P$ - Pressão total $(P a)$;

$R$ - constante universal dos gases $\left(\mathrm{J} \mathrm{mol}^{-1} \mathrm{~K}^{-1}\right)$;

$R_{j}$ - taxa cinética da reação $j\left(\mathrm{~mol} \mathrm{~m}^{-3} \mathrm{~s}^{-1}\right)$;

$\rho$ - massa específica da mistura reacional $\left(\mathrm{kg} \mathrm{m}^{-3}\right)$;

$T$ - Temperatura $(K)$;

$t^{\prime}$ - tempo real $(s)$;

$t$ - tempo adimensional;

$v$ - velocidade axial $\left(m \cdot s^{-1}\right)$

$z$ - posição axial do leito $(m)$.

\section{REFERÊNCIAS}

ÁVILA NETO, C. N. Análise termodinâmica das reformas do metano, do etanol e da nafta. Dissertação de Mestrado - Faculdade de Engenharia Química, Universidade Federal de Uberlândia, Uberlândia, 2009.

BISCHOFF, K. B. A note on boundary conditions for flow reactors. Chem. Eng. Sci., v. 16, p. 131-133, 1961.

DANCKWERTS, P. V. Continuous flow systems. Distribution of residence times. Chem. Eng. Sci., v.1, p. 1-13, 1953.

FORNBERG, B. Calculation of weights in finite difference formulas. Soc. Ind. App. Math., v.40, n. 3, p. 685-691, 1998.

KAGYRMANOVA, A.P;. CHUMACHENKO, V.A, KOROTKIKH, V.N; KASHKIN, V.N; NOSKOV, A.S. Catalytic dehydration of bioethanol to ethylene: Pilot-scale studies and process simulation. Chem. Eng. J., v. 176-177, p. 188-194, 2011.

LAGMUIR, I. The velocity of reactions in gases moving through heated vessels and the effect of convection and diffusion. J. Amer. Chem. Soc. v. 30, p. 1742-1754, 1908.

MORSCHBACKER, A. Bio-ethanol based ethylene. Pol. Rev., v. 49, n. 2, p. 79-84, 2009. 